\title{
Fibroblast activation protein augments progression and metastasis of pancreatic ductal adenocarcinoma
}

\author{
Albert Lo, ${ }^{1,2}$ Chung-Pin Li, ${ }^{3,4}$ Elizabeth L. Buza, ${ }^{5}$ Rachel Blomberg, ${ }^{1}$ Priya Govindaraju, ${ }^{1}$ Diana Avery, \\ James Monslow, ${ }^{1}$ Michael Hsiao, ${ }^{6}$ and Ellen Puré ${ }^{1}$ \\ 'Department of Biomedical Sciences and '2Cell and Molecular Biology Graduate Group, University of Pennsylvania, \\ Philadelphia, Pennsylvania, USA. ${ }^{3}$ Division of Gastroenterology and Hepatology, Department of Medicine, Taipei Veterans \\ General Hospital, Taipei, Taiwan. ${ }^{4}$ National Yang-Ming University School of Medicine, Taipei, Taiwan. ${ }^{5}$ Department of \\ Pathobiology, University of Pennsylvania, Philadelphia, Pennsylvania, USA. ${ }^{6}$ Genomics Research Center, Academia Sinica, \\ Taipei, Taiwan.
}

Pancreatic ductal adenocarcinomas (PDAs) are desmoplastic and can undergo epithelial-tomesenchymal transition to confer metastasis and chemoresistance. Studies have demonstrated that phenotypically and functionally distinct stromal cell populations exist in PDAs. Fibroblast activation protein-expressing (FAP-expressing) cells act to enhance PDA progression, while $\alpha$ smooth muscle actin myofibroblasts can restrain PDA. Thus, identification of precise molecular targets that mediate the protumorigenic activity of $\mathrm{FAP}^{+}$cells will guide development of therapy for PDA. Herein, we demonstrate that FAP overexpression in the tumor microenvironment correlates with poor overall and disease-free survival of PDA patients. Genetic deletion of FAP delayed onset of primary tumor and prolonged survival of mice in the KPC mouse model of PDA. While genetic deletion of FAP did not affect primary tumor weight in advanced disease, FAP deficiency increased tumor necrosis and impeded metastasis to multiple organs. Lineage-tracing studies unexpectedly showed that FAP is not only expressed by stromal cells, but can also be detected in a subset of $\mathrm{CD} \mathrm{O}^{+}$mesenchymal PDA cells, representing up to $20 \%$ of total intratumoral FAP ${ }^{+}$cells. These data suggest that FAP may regulate PDA progression and metastasis in cell-autonomous and/or non-cell-autonomous fashions. Together, these data support pursuing FAP as a therapeutic target in PDA.

Conflict of interest: The authors have declared that no conflict of interest exists.

Submitted: December 16, 2016

Accepted: August 24, 2017

Published: October 5, 2017

Reference information: JCI Insight. 2017;2(19):e92232. https://doi.org/10.1172/jci. insight. 92232

\section{Introduction}

Pancreatic ductal adenocarcinoma (PDA) is a lethal disease refractory to existing therapies. The outcome of these patients remains dismal, with a median survival of less than 6 months and a 5 -year survival rate of less than $5 \%$ (1). Given the high mutation rate and the importance of KRAS and p53 signaling pathways in regulating cell proliferation and survival, a genetically engineered mouse model of pancreatic cancer, referred to as KPC mice, was developed using Cre-loxP recombination to drive expression of oncogenic Kras ${ }^{(\mathrm{KrasG} 12 \mathrm{D})}$ and mutant Trp53 ${ }^{(\mathrm{Tr} p 53 \mathrm{R} 172 \mathrm{H})}$. KPC mice stochastically form preneoplastic pancreatic lesions that eventually progress to invasive PDA with $100 \%$ penetrance (2). This autochthonous mouse model of pancreatic cancer faithfully recapitulates the clinical manifestations, histological features, metastatic profiles, chemoresistance, and proinflammatory and immunologically tolerant hallmarks of PDA (3), making it a relevant preclinical model for studying pancreatic cancer biology and testing novel therapeutics.

Fibroblast activation protein (FAP) is a type II transmembrane cell surface proteinase (4). FAP belongs to the prolyl dipeptidyl aminopeptidase (DPP) family, which cleaves amino-terminal dipeptides from polypeptides with proline or alanine in the penultimate position (P1Pro or P1Ala) (5). FAP also exhibits endopeptidase activity that preferentially cleaves after the Gly-Pro sequence motif (P2GlyP1Pro) (6). Overexpression of FAP has been noted in both malignant tumor cells and stromal fibroblasts (7-9). Studies have demonstrated that FAP protease activity is required in the processing of cytokines, chemokines, and extracellular matrix components and that it shapes the secretome and proteome of cancer-associated fibroblasts (CAFs) (10-13). Additionally, although FAP only has a short cytoplasmic tail, recent studies 
A

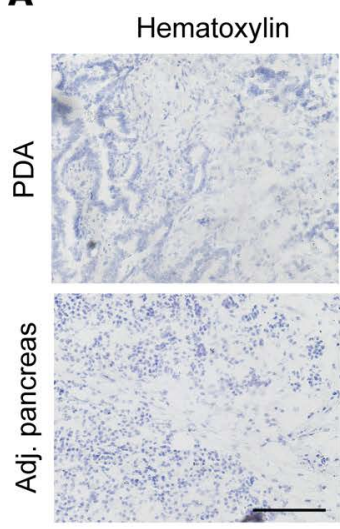

Case 1
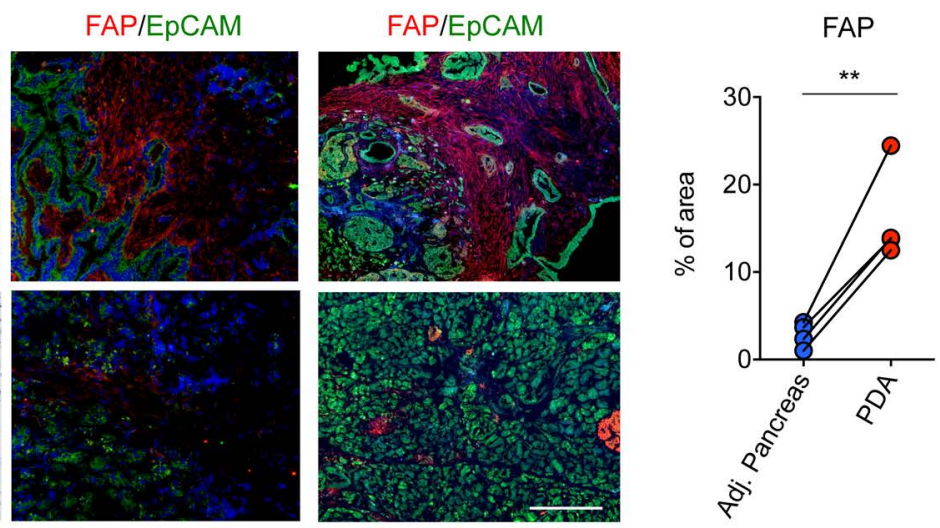

B

Pancreas (sham surgery)

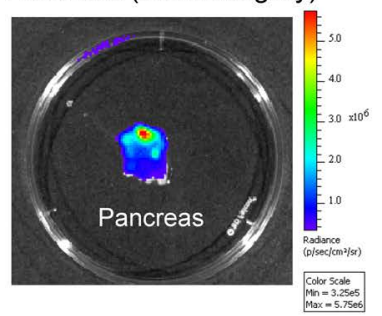

Orthotopic PDA

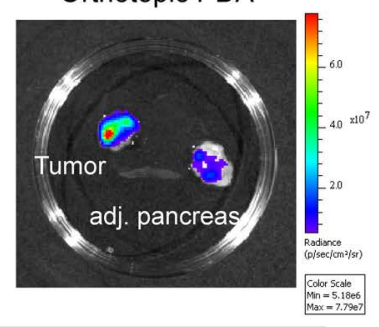

FAP-luciferase reporter mice (FAPluc/luc)

isotype

C

FAP

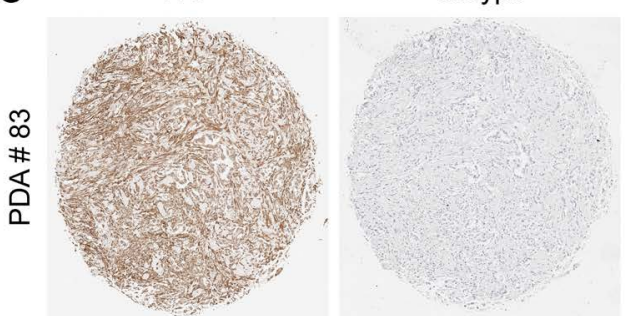

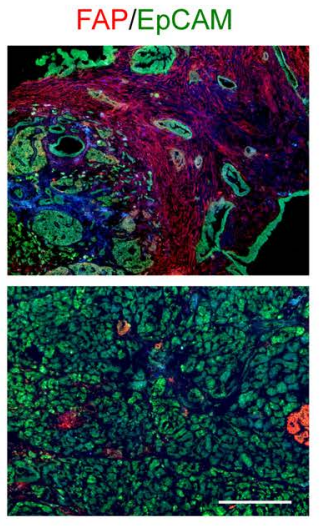

Case 2

Orthotopic PDA

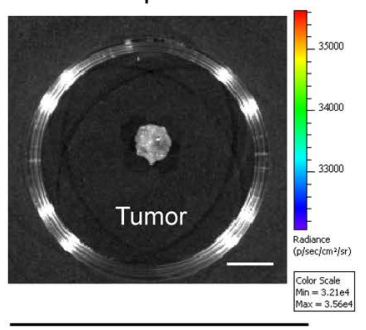

WT C57BL/6 mice

Figure 1. FAP is overexpressed in human and mouse pancreatic ductal carcinomas. (A) Human PDA and adjacent normal pancreatic tissues were subjected to hematoxylin and immunofluorescent staining with FAP and EpCAM. Scale bar: $100 \mu \mathrm{m}$. FAP is highly expressed across the whole tumor tissue compared with the adjacent normal pancreas $(n=4)$. ${ }^{*} P<0.01$ by Student's $t$ test. (B) FAP luciferase reporter knockin or WT C57BL/6 mice were orthotopically implanted with syngeneic Pan02 PDA cells or serum-free medium (sham surgery) in the tail of the pancreas $(n=2)$. Tumor and adjacent pancreatic tissues were harvested and subjected to bioluminescent imaging. Scale bar: 1 $\mathrm{cm}$. (C) Analysis of FAP expression using human PDA tissue microarray. Scale bar: $400 \mu \mathrm{m}$. PDA, pancreatic ductal carcinomas.

have revealed that FAP can also regulate signal transduction pathways and malignant tumor behavior in a cell-autonomous fashion $(7,14)$. We previously showed that genetic deletion of FAP and pharmacological inhibition of FAP protease activity reduced the growth of mutant Kras-induced primary lung cancers and tumor growth in a syngeneic transplant model of colon cancer (9). Tumor inhibition induced by FAP deficiency was attributed to decreased tumor angiogenesis and altered ECM remodeling. Nonetheless, the effect of FAP on other tumor types cannot merely be extrapolated from these results; as was found in melanoma, FAP has, in contrast, been reported to restrain tumor growth (15). The roles of FAP in pancreatic cancer, one of the most desmoplastic and aggressive tumor types, are not known, and its effect on metastasis of any tumor type has not yet been explored. In this report, we provide evidence that FAP expression is an independent predictor of outcome in human PDA and describe the antitumorigenic effect of FAP deletion on tumorigenesis, progression, and metastasis in the autochthonous KPC mouse model of PDA.

\section{Results}

Overexpression of FAP correlates with poor clinical outcome in human PDA. To determine whether FAP might be a prognostic indicator or therapeutic target in PDA, we first examined the expression of FAP in clinical specimens. Immunofluorescent staining showed that FAP was overexpressed by stromal cells in PDAs and that, in comparison, expression of FAP was markedly reduced in adjacent pancreatic tissue (Figure 1A). This selective expression pattern was confirmed using Fap luciferase reporter mice, in which low basal levels of FAP expression were detected in normal pancreatic tissue but markedly upregulated in pancreatic 
A
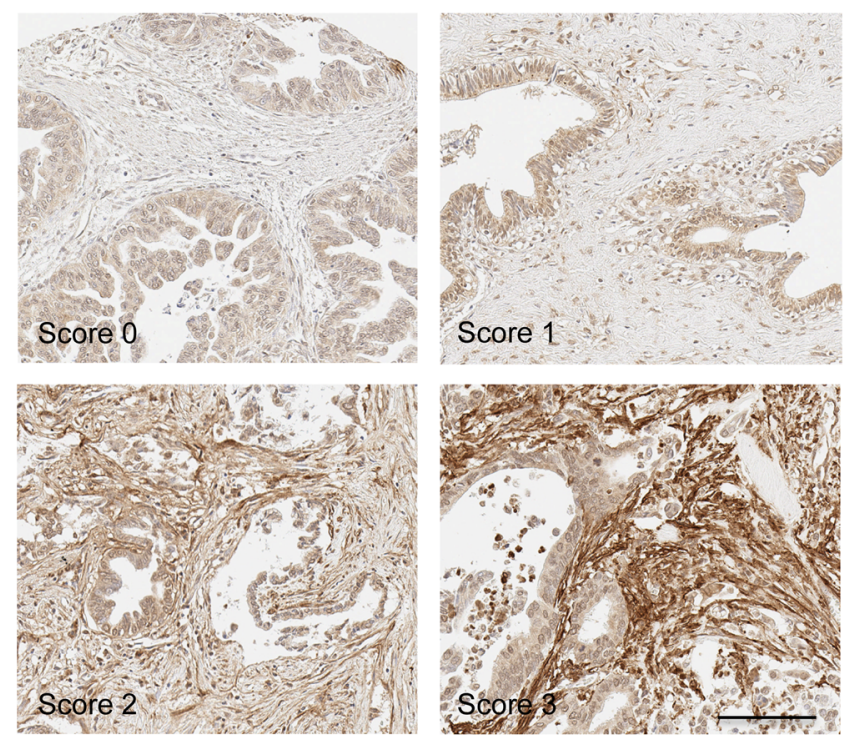

B

Overall survival

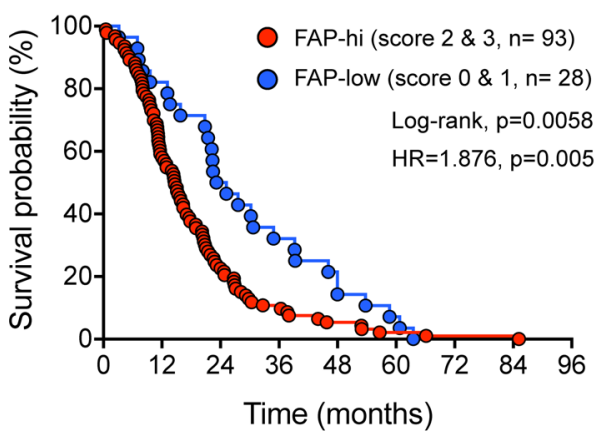

Disease-free survival

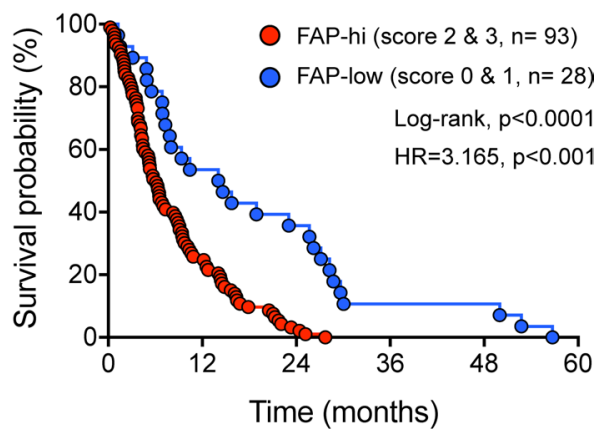

Figure 2. FAP expression levels correlate with poor clinical outcome in human PDA. (A) Representative images of FAP expression scores in PDA. The scores are determined based on the intensity and prevalence of FAP staining in the tumor stroma. Scale bar: $100 \mu \mathrm{m}$. (B) Kaplan-Meier analysis of overall survival and disease-free survival of 121 patients with PDA stratified by FAP expression level. Significance was determined using the Mantel-Cox (log-rank) test. Multivariate Cox regression analysis indicates higher hazard ratio (HR) for risk of death in patients with PDA in both overall survival and disease-free survival, $P<0.01$ and $P<$ 0.001 , respectively.

tumors derived from the PanO2 PDA cell line transplanted orthotopically into syngeneic mice (Figure 1B). Immunostaining of tissue microarrays (TMAs) containing primary PDAs from 121 patients was then performed. The clinicopathologic information regarding patient demographics, pathologic tumor stage, and survival times is provided in Supplemental Table 1 (supplemental material available online with this article; https://doi.org/10.1172/jci.insight.92232DS1). FAP was highly expressed in human PDA specimens (Figure 1C). FAP expression was further scored according to staining intensity and prevalence on a scale from 0 to 3 (Figure 2A). Using this defined scoring criteria, we stratified patients into FAP-high (scores of 2 and 3 ) and FAP-low groups (scores of 0 and 1). No association was found between FAP expression level and stage, tumor size, or regional or distal lymph node metastasis (Supplemental Table 2). However, high levels of FAP expression were associated with shorter overall survival and disease-free survival compared with patients with low FAP expression (Figure 2B). Univariate regression and multiple regression analyses of overall survival and disease-free survival indicated that FAP expression level was a significant, independent predictor of survival for patients with PDA (Supplemental Table 3).

FAP accelerates tumor progression and decreases survival in a mouse model of PDA. We previously reported that FAP deficiency impaired tumor initiation and progression in mutant Kras-driven lung tumors and a syngeneic transplant model of colon cancer (9); in view of the lack of effective therapies and the typically high stromal cell content that has been implicated in therapy resistance of PDAs, it was of particular interest to determine whether FAP also promotes tumorigenesis in the context of PDA. Using the KPC mouse model of PDA, we tested the effect of FAP deletion on tumor initiation, progression, and metastasis. We first confirmed that FAP deletion had no apparent effect on pancreatic development, as serum glucose and amylase levels, as well as pancreata weight, morphology, and histologic features, showed no difference (Supplemental Figure 1). Additionally, expression of FAP by stromal cells is haplosufficient, and genetic 
A

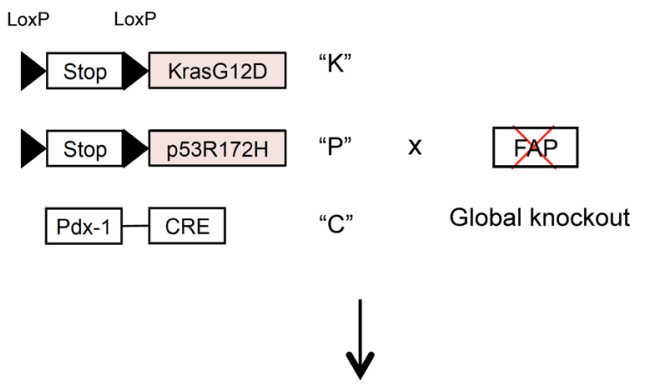

C

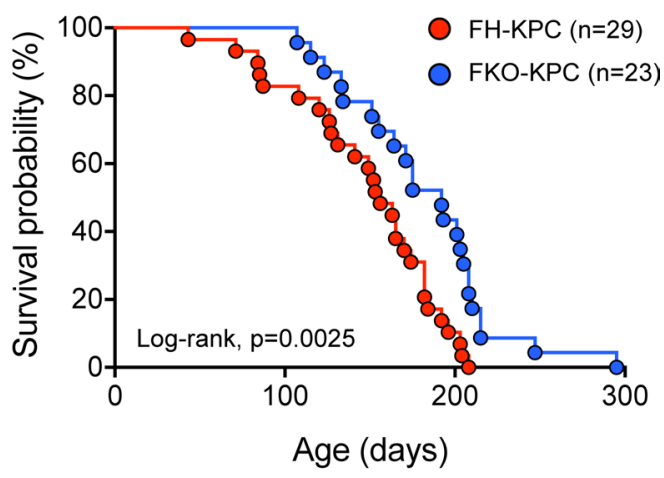

Figure 3. Genetic targeting of FAP delays tumor progression and prolongs the survival in a preclinical mouse model of pancreatic cancer.

(A) Schematic of the mouse model used in this study. LSL-Kras ${ }^{\mathrm{C12D/+}}$, LSL-Trp53 $3^{\mathrm{R} 172 \mathrm{H}}$, and $\mathrm{Pdx}-1$-Cre (KPC) mice were crossed with FAP-deficient mice to generate FAP-heterozygous KPC (FH-KPC) and global FAP-deficient KPC (FKO-KPC) mice. Triangles flanking stop sites in $\mathrm{K}$ and $\mathrm{P}$ indicate LoxP recombination sites. (B) Tumor detection analysis. Mice underwent sonographic examinations to evaluate for the development of PDAs (FH-KPC, $n=29$; FKO-KPC, $n=23) . P<0.001$ by Mantel-Cox (log-rank) test. (C) Kaplan-Meier survival analysis. Mice that developed severe ulcerated facial or vaginal papillomas or large thymic lymphomas were not included in the survival study. $P<0.005$ by Mantel-Cox (log-rank) test.

deletion of FAP did not ablate tumor stromal cells in pancreatic tumors (Supplemental Figure 2). Based on these findings, Global FAP-knockout mice were crossed with KPC mice to generate FAP-heterozygous KPC (FH-KPC) and FAP-knockout KPC (FKO-KPC) mice (Figure 3A). Both genotypes were born at expected Mendelian ratios and appeared phenotypically normal at birth. PDAs arose in both FH-KPC and FKO-KPC mice, with comparable tumor volume at first detection and similar numbers of tumors between the two groups (Supplemental Figure 3, A and B). Thus, FAP is not essential for PDA tumorigenesis driven by oncogenic Kras in this model. Analysis of sonographic data revealed, however, that FAP deficiency delayed the onset of PDA, shifting the median time to tumor detection from 16.4 weeks to 21.9 weeks $(P<0.001)$ (Figure 3B). Moreover, genetic deletion of FAP prolonged the survival of KPC mice. FKO-KPC mice had a median survival of 192 days, compared with a median survival of 156 days for FH-KPC mice $(P<0.005)$ (Figure $3 C)$. Additionally, subcutaneous transplantation of syngeneic KPC tumor cells into mice showed that FAP deficiency delayed their initial growth (Figure 4). Taken together, these data indicate that FAP may accelerate, but is not required for, the initiation of PDA development and plays a critical role in promoting disease progression.

FAP confers resistance to necrotic cell death and drives PDA metastasis to multiple visceral organs. Undifferentiated histologies are indicative of highly aggressive and lethal tumors in the setting of PDA $(16,17)$. To determine if the delayed onset of PDAs in the FKO-KPC mice is associated with altered tumor histology, we performed histopathological analyses to assess the composition of the PDAs. Targeting FAP did not effect the distribution 


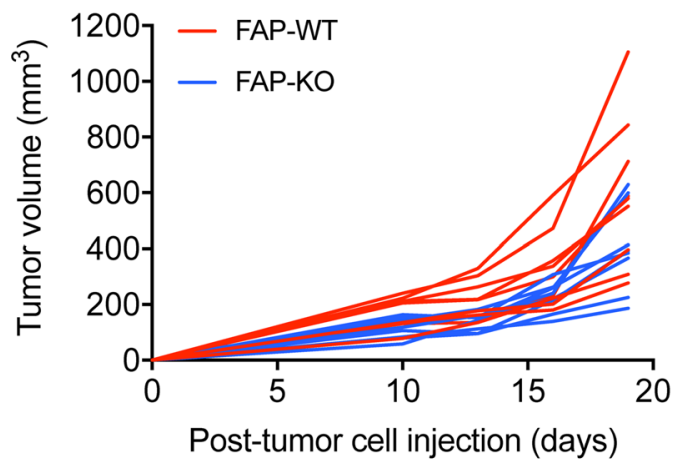

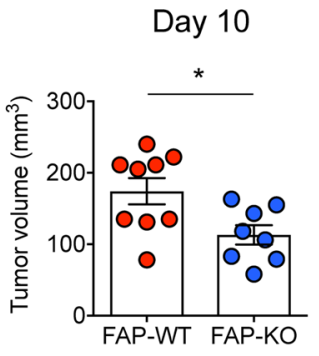

Day 16

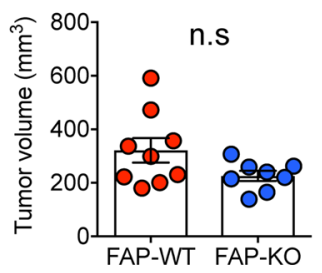

Day 13

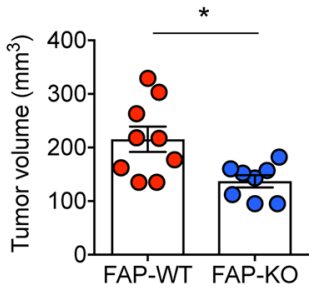

Day 19

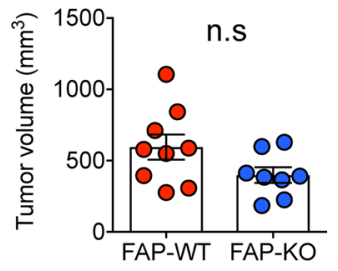

Figure 4. Targeting FAP delays the growth of subcutaneously transplanted syngeneic KPC tumor cells. Syngeneic KPC tumor cells were implanted subcutaneously in FAP-WT and FAP-KO mice ( $n=8 \sim 9$ per group). Tumor growth was measured by electronic caliper and plotted individually. Results are shown as mean $\pm \mathrm{SEM}$. ${ }^{*} P<0.05$ by Student's $t$ test.

of PDA subtypes, as about $80 \%$ of the tumors in both FH-KPC and FKO-KPC mice exhibited primary ductal structure, while the remainder showed undifferentiated phenotypes (Supplemental Figure 3C). No difference was found in the differentiation status of glandular tumor structure between FH-KPC and FKO-KPC mice (Supplemental Figure 3D). Interestingly, sonographic analyses revealed that the PDAs that developed in FKOKPC mice exhibited marked hypoechoic area, an indication of tumor necrosis, relative to the PDAs developed in FH-KPC mice (Figure 5A). Further histopathological studies of the tumors from the two groups confirmed this finding (Figure 5B), demonstrating a significant correlation between FAP deficiency and tumor necrosis $(P$ $=0.011$ ). We also noted that the increase in necrotic tumor cell death in tumors from FAP-deficient mice was associated with increased lymphocytic cell infiltration, suggesting that FAP expression may contribute to the resistance of PDAs to immune-mediated control of tumor progression.

To determine whether FAP is essential for PDA metastasis, we examined the metastatic lesions in multiple visceral organs. At the time of sacrifice (moribund mice), primary tumor weights between the FH-KPC and FKO-KPC mice were not different (Figure 6A). However, the frequency of PDA metastasis in liver, lung, mesentery, and diaphragm/peritoneum was decreased in FKO-KPC mice $(P<0.05)$, indicating that FAP enhances tumor metastasis (Figure 6B). In addition, the frequency of both liver and pulmonary macrometastatic lesions identified at the time of necropsy was reduced in FKO-KPC mice (Figure $6 \mathrm{C})$, suggesting that FAP may also drive metastatic outgrowth.

Lineage-tracing studies were performed to determine the source of FAP-expressing cells in PDA using KPC-Rosa ${ }^{\mathrm{YFP}}$ mice (18) (Figure 7A). Flow cytometric analysis of the primary tumors from this model revealed that FAP was not only expressed by the stromal cells ( $\mathrm{YFP}^{-}$), but also could be upregulated in $80 \%$ of $\mathrm{CD}^{-} 5^{-} \mathrm{CD} 90^{+}$mesenchymal-like PDA cells, which represented $4.4 \%$ of total $\mathrm{YFP}^{+} \mathrm{PDA}$ cells (Figure 7B). This population represents $20 \%$ of total $\mathrm{FAP}^{+}$cells in $\mathrm{KPC}$ tumors (Figure $7 \mathrm{C}$ ). Collectively, these data indicate that FAP protease expressed by stromal and/or tumor cells may play critical roles in the progression and metastasis of pancreatic cancer.

\section{Discussion}

Pancreatic cancer, characterized by an extensive desmoplastic stromal reaction, represents one of greatest challenges in oncology. Stromal elements are prevalent in clinical specimens and can predict poor prognostic outcomes (19). Earlier studies employing in vitro culture or in vivo transplantation approaches have demonstrated that various stromal elements can promote PDA proliferation, progression, and metastasis (20-22). Recent studies, however, have revealed that cancer-associated stromal cells, including CAFs, are phenotypically heterogeneous and, at the cellular level, can have opposing effects, in particular, in the 
A
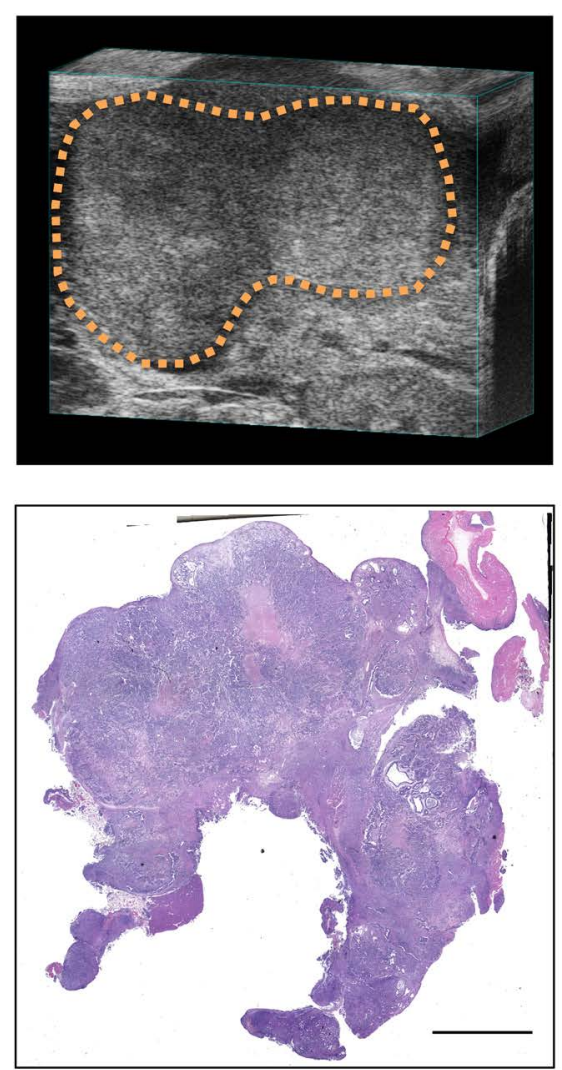

B

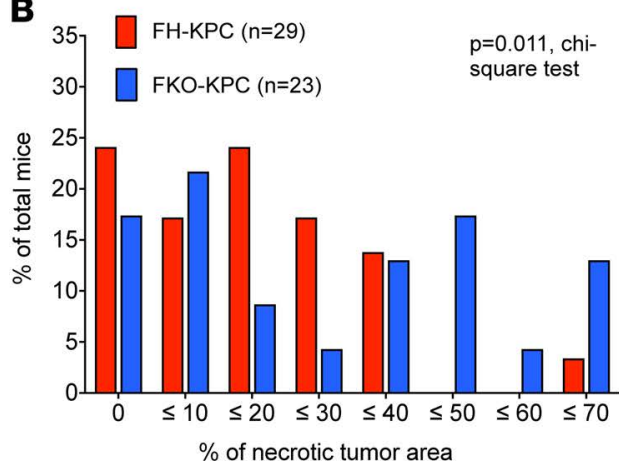

FKO-KPC
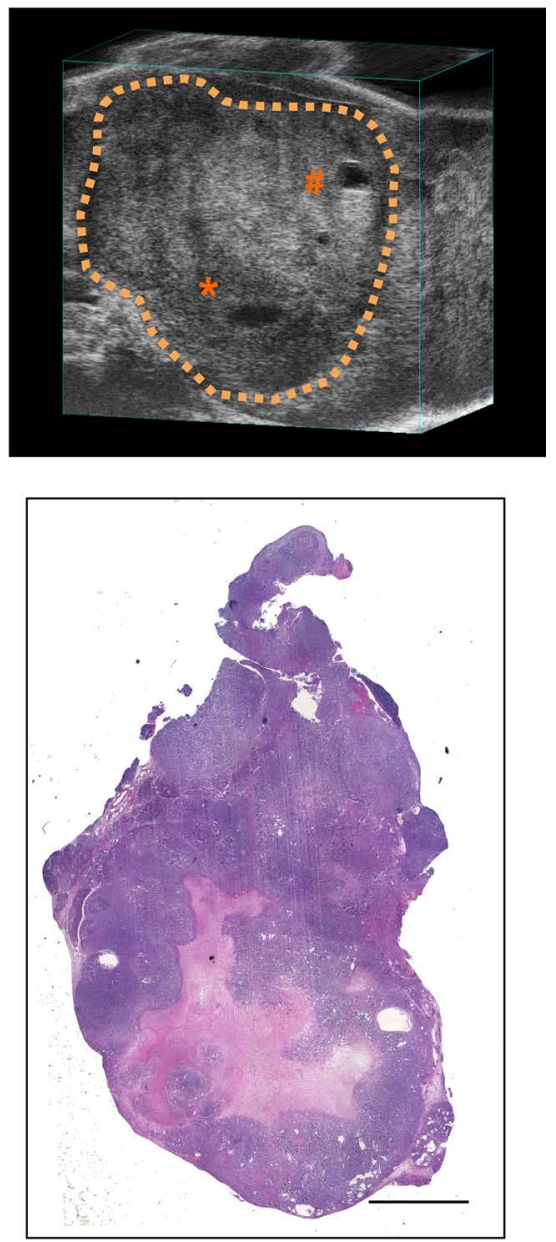

Figure 5. Targeting FAP alters internal tumor architecture associated with necrotic cell death. (A) Representative ultrasound images of the pancreatic tumors. The dashed line shows the border of pancreatic tumors. Within the tumor, the hypoechoic (darker) area is indicative of tumor necrosis, whereas hyperechoic (brighter) regions represent heterogeneous tumor structure, including stroma and vessels. Extensive tumor necrosis and a small cyst within the tumor are denoted using an asterisk and a number sign, respectively. (B) Tumors were harvested at the endpoint and stained with H\&E (FH-KPC, $n=29$; FKOKPC, $n=23)$. Scale bar: $0.5 \mathrm{~cm}$. The degree of necrosis within each tumor was assessed by a board-certified pathologist (ELB). $\chi^{2}$ test shows a statistically significant correlation between tumor necrosis and FAP deletion.

context of PDA. Specifically, deletion of $\mathrm{FAP}^{+}$cells that include the majority of CAFs and a subset of M2-like macrophages, using a FAP-targeted immune-based therapeutic approach (23) or a genetic ablation approach $(8,24)$, inhibited PDA growth. In contrast, ablation of $\mathrm{SMA}^{+}$cells (i.e., myofibroblasts) led to the development of more invasive, undifferentiated tumors with enhanced hypoxia, epithelial-to-mesenchymal transition (EMT), and cancer stem cells, with diminished animal survival (16). This evidence for complexity in the complicity of stromal cells in preclinical PDA models is consistent with the association of high levels of FAP and SMA with reduced and increased survival of PDA patients, respectively $(16,25)$. At a molecular level, tumor cell expression of sonic hedgehog (Shh) drives the formation of a fibroblast-rich desmoplastic stroma. As predicted, PDAs in Shh-deficient KPC mice exhibited reduced stromal content but were more aggressive, undifferentiated, and highly proliferative as well as exhibiting increased angiogenesis that was associated with reduced survival (17). In contrast, in this study, we show that overexpression of FAP is associated with worse clinical outcome in PDA patients, demonstrate that FAP is crucial for progression and metastasis in an autochthonous mouse model of PDA, and, therefore, suggest that it may represent a therapeutic target in PDA. Collectively, these results highlight the complexity of the tumor stroma interaction and provide evidence that, at both the cellular level and at a molecular 
A

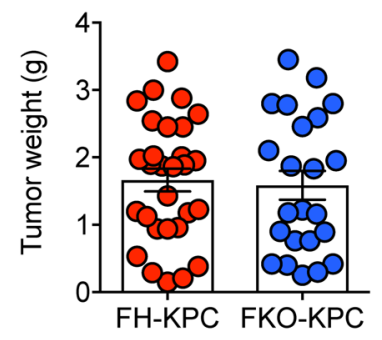

B

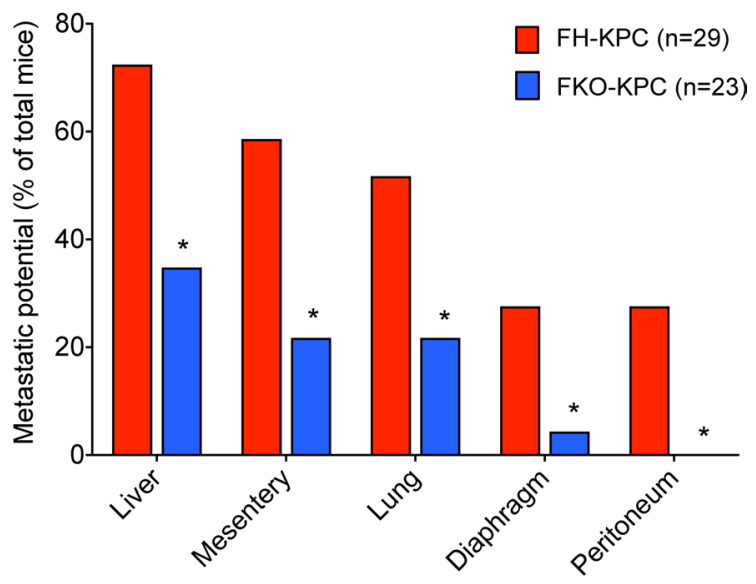

Figure 6. Targeting FAP impedes pancreatic cancer metastasis. (A) Weights of primary tumors at the endpoint. Results are shown as mean \pm SEM (FH-KPC, $n=29$; FKO-KPC, $n=23$ ). (B) Metastases in multiple organs were evaluated at necropsy and confirmed by H\&E staining. ${ }^{*} P<0.05$ by Fisher's exact test. (C). Fractions of mice with macroscopic lesions by visual inspection at the time of necropsy and microscopic lesions identified by H\&E staining from liver and lung are shown. Scale bar: $100 \mu \mathrm{m}$.
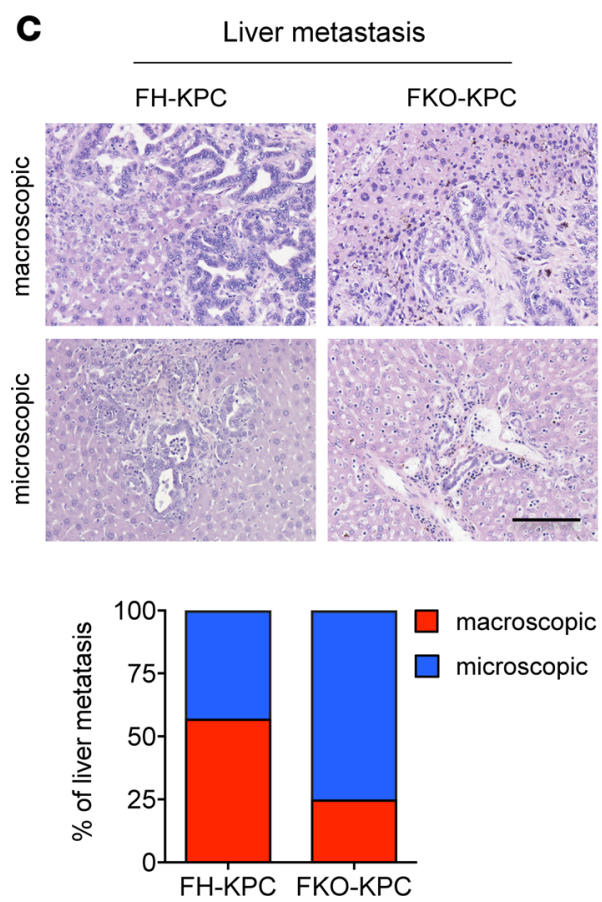
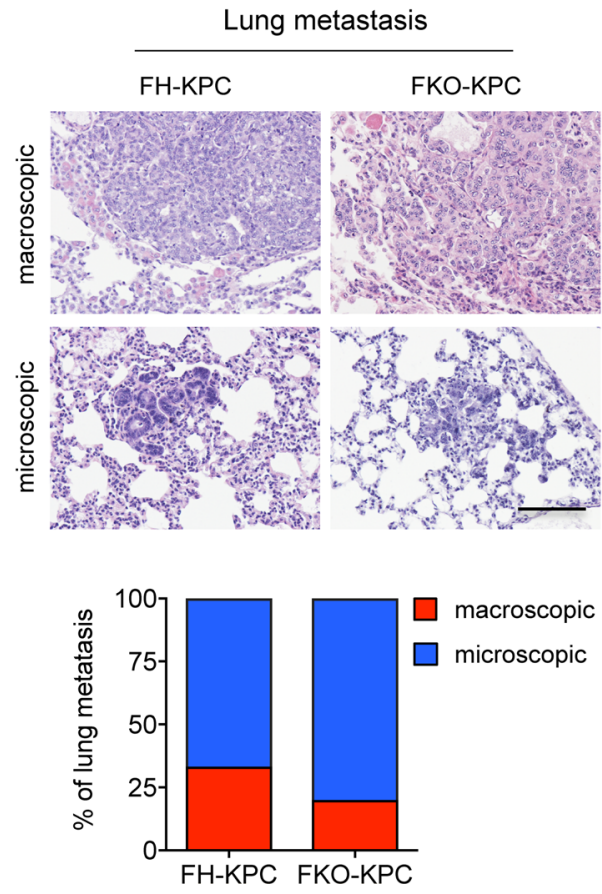

level, stromal cells can have opposing effects on the development and progression of PDA. This complexity must be taken into careful consideration in designing potential stromal cell-targeted therapeutic approaches to the treatment of pancreatic cancer.

In primary PDAs, deposition of extracellular matrix elements, such as collagen and hyaluronan, was shown to negatively correlate with patient survival. Moreover, both primary tumors and metastases of PDA exhibit stromal fibrosis (19). These observations indicate that the desmoplastic response may effect both progression of the primary tumor as well as distant metastasis. Interestingly, FAP protease-mediated remodeling of the tumor stroma was shown to promote tumor cell migration (26). Coculture of $3 \mathrm{~T} 3$ fibroblasts forced to express FAP was demonstrated to increase invasive ability of pancreatic cancer cells (27). Collectively, these data suggest that FAP may regulate early steps of the metastasis cascade, i.e., tumor cell dissemination and/or invasion. This may in part explain our observation that the frequency of PDA metastasis to multiple visceral organs is decreased in FKO-KPC mice. Future studies utilizing three-dimensional organotypic culture with fibroblasts or fibroblast-derived matrix may determine whether FAP is essential for PDA invasion and dissemination. Another, not mutually exclusive explanation is that FAP is involved in the formation of a premetastatic niche. It will therefore be interesting to examine FAP expression levels in metastatic organs at the preneoplastic stage and determine whether targeting FAP will alter matrix modeling, thereby inhibiting malignant cell seeding. 
A

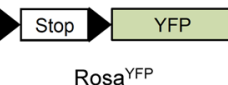

RosaYFP

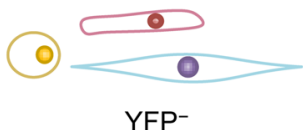

(hematopoietic cells, fibroblasts, pericytes ...etc)

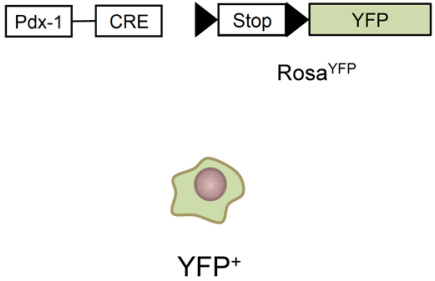

(pancreatic epithelial-derived cells)
B

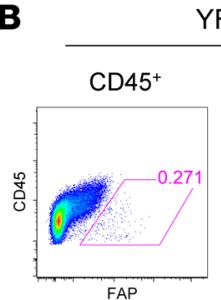

YFP-
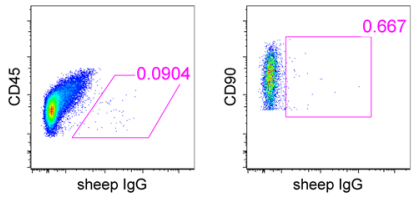

C FAP-expressing cells

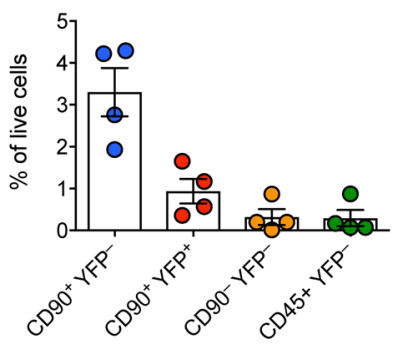

YFP+
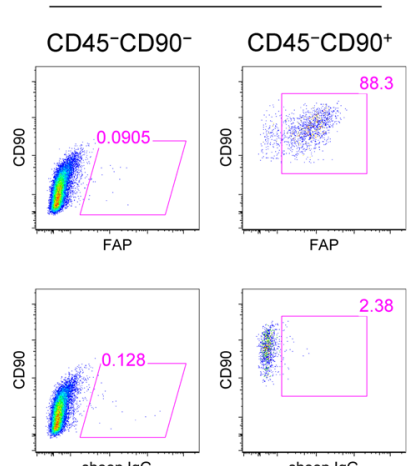

sheep $\lg G$

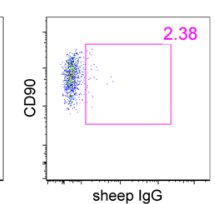

Normalization
$\mathrm{CD}^{-5^{-} \text {YFP }^{+}}$
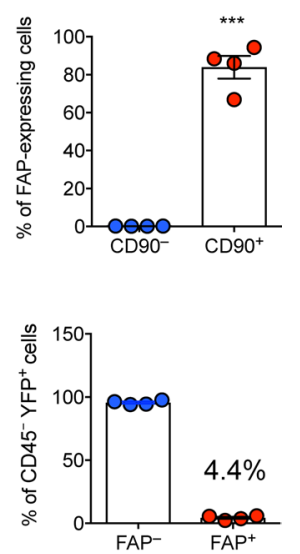

Figure 7. Lineage tracing reveals that stromal cells and a subset of mesenchymal-like pancreatic cancer cells express FAP. (A) Schematic of the KPC-Rosa ${ }^{\mathrm{YFP}}$ mouse model used for characterizing FAP-expressing cells. Cre-mediated recombination of the lineage label renders tumor cells $\mathrm{YFP}^{+}$, while rest of the stromal cells are YFP. (B) Established pancreatic tumors were harvested from KPC-Rosa ${ }^{\mathrm{YFP}}$ mice and sorted by YFP for flow cytometry analysis. Both $\mathrm{CD} 45^{+}$hematopoietic cells and $\mathrm{CD} 45^{-} \mathrm{CD} 90^{+}$stromal cells express FAP. $A$ fraction of $\mathrm{YFP}^{+}$tumor cells upregulate mesenchymal stromal cell markers CD90 and FAP. Results are shown as mean \pm SEM $(n=4) .{ }^{* *} P<0.001$ by Student's $t$ test. (C) Quantification and normalization of total FAP-expressing cells in pancreatic tumors. Results are shown as mean \pm SEM.

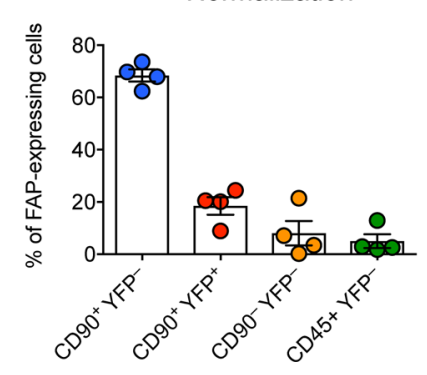

Furthermore, macroscopic liver and pulmonary metastases were less frequent in FKO-KPC mice, suggesting that FAP may drive the outgrowth of metastatic lesions. Preliminary findings from our ongoing studies suggest that disruption of FAP protease inhibits pulmonary metastatic outgrowth and that this inhibition correlates with reduced macrophage infiltration (A. Lo et al., unpublished observations), which may be due to downregulation of CCL2 from FAP-deficient CAFs $(12,13)$. Further studies are required to determine if this mechanism can be extended to PDA metastasis. Another interesting possibility is that FAP expression in mesenchymal-like cancer cells may promote invasion or metastasis in a cell-autonomous fashion. Reports demonstrating that FAP can regulate PTEN/PI3K/AKT and Ras/ERK signaling pathways to enhance tumor cells undergoing EMT, migration, invasion, and metastasis support this hypothesis $(7,14)$. Upregulation of FAP in PDA cells undergoing EMT may offer an opportunity to target these cells therapeutically with chimeric antigen receptor $\mathrm{T}$ cells as previously reported $(23,28-31)$. Careful examination and dissection of the antitumor effect achieved by targeting $\mathrm{FAP}^{+}$stromal cells and/or $\mathrm{FAP}^{+} \mathrm{EMT}^{-}$ tumor cells would be critical in future studies.

Finally, production of SDF-1 $\alpha /$ CXCL12 by $\mathrm{FAP}^{+}$stromal cells was implicated in mediating $\mathrm{T}$ cell exclusion through an unknown mechanism (24). Prior studies revealed that FAP can mediate proteolytic processing of SDF-1 $\alpha$ /CXCL12 by its DPP activity (10). Truncated SDF-1 $\alpha /$ CXCL12 was shown to act as an antagonist, resulting in loss of both lymphocyte-chemotactic and CXCR4-signaling properties (32). Based on these observations, it will be interesting to address whether disruption of FAP protease activity may permit $\mathrm{T}$ cell recruitment and infiltration, thereby causing heightened necrotic death found in FAP-deficient PDAs. 
In summary, the results presented herein indicate that heightened FAP expression is an independent prognostic factor for human PDA. We provide genetic evidence that FAP plays key roles in pancreatic cancer progression and metastasis and may serve as a candidate target in treating this devastating disease.

\section{Methods}

Animals. FAP-luciferase reporter mice $\left(F a p^{\text {Luc/Luc }}\right)$ and FAP-deficient mice (Fap $\left.{ }^{\text {DTR/DTR }}\right)$ were generated by modifying a targeting construct provided by Andreas Schnapp at Boehringer-Ingelheim. Kras $^{L S L-G 12 D /+}$ and Pdx-1-Cre C57BL/ 6 mice were provided by Sandra Ryeom and Robert Vonderheide, respectively, at the University of Pennsylvania. Trp53 $3^{L S L-R 172 H /+}$ mice on a mixed 129/SvJae/C57BL/6 background were acquired from the Mouse Repository at the National Cancer Institute. Mice were backcrossed at least 8 generations onto a C57BL/ 6 genetic background and then interbred to generate Kras $^{\text {G12D. }}$ Trp53 ${ }^{R 172 H}:$ Pdx-1-Cre (KPC) mice.

Cell culture. Murine primary PDA cells were derived from a pancreatic tumor isolated from a fully backcrossed KPC mouse, recapitulating the desmoplastic feature of autochthonous PDA (23). Pan02 murine PDA cells were obtained from the National Cancer Institute Developmental Therapeutics Program Tumor Repository. Mouse adult lung fibroblasts were isolated from 8- to 12-week-old FAP-WT and FAP-KO C57BL/6 mice. Cells were authenticated by morphology, growth characteristics, and biologic behavior; tested to confirm that cells were mycoplasma free; and frozen. Cells were cultured in RPMI supplemented with $10 \%$ FBS and $1 \%$ penicillin-streptomycin and used less than 1 month after resuscitation.

Bioluminescence imaging. The IVIS Lumina III Bioluminescence and Fluorescence Imaging System (PerkinElmer) was used for in vivo bioluminescent imaging. Pancreatic tumor-bearing mice and animals that underwent sham surgery were injected intraperitoneally with $150 \mathrm{mg} / \mathrm{kg}$ body D-luciferin (Gold Biotechnology). Bioluminescent signal from FAP-expressing cells was quantified by Living Image software (PerkinElmer).

Ultrasound imaging. High-resolution sonography was performed using the Vevo 2100 imaging system (FUJIFILM VisualSonics). Mice underwent weekly ultrasound scans beginning at 8-10 weeks of age to detect the presence of pancreatic tumors and/or metastatic disease. Tumors were digitally visualized and reconstructed for measuring tumor volume using the Integrated Vevo Workstation software package (FUJIFILM VisualSonics). Mice becoming moribund or showing signs of distress, such as anorexia and cachexia, due to tumor burden were considered to have reached the study endpoint. Mice were not included in the survival study if they developed severe ulcerated facial or vaginal papillomas or exhibited large thymic lymphomas.

Patient samples and TMA. PDA samples were obtained from Taipei Veterans General Hospital Tissue Banks. The TMAs were constructed with 1.0-mm-sized tumor and normal tissues extracted from paraffin donor blocks. Four- $\mu \mathrm{m}$ sections were cut from the TMA blocks, and three independent sections from three TMAs were used for immunohistochemical staining.

Histopathological, immunohistochemical, and immunofluorescent analysis. TMA IHC staining was performed by using the Discovery XT automated immunostainer (Ventana Medical Systems Inc.). Heat-induced antigen retrieval was performed in Tris-EDTA buffer. The slides were stained with polyclonal sheep FAP antibody (R\&D Systems, catalog AF3715) or isotype-matched control (R\&D Systems, catalog 5-001-A). FAP expression was scored according to staining intensity and prevalence on a scale from 0-3: 0 , no staining; 1, weak staining; 2, moderate staining; 3 , strong staining. Mouse pancreas, pancreatic tumors, and visceral organs were harvested, fixed with Prefer (Anatech), and paraffin embedded. Tissue sections were stained with Masson's trichrome, Alcian blue, or H\&E. For immunofluorescent staining, frozen sections were fixed in acetone and stained with F19-FAP antibody and EpCAM antibody (Cell Signaling, catalog 5198).

Flow cytometric analyses. Single-cell suspensions were prepared from primary skin fibroblast culture or murine PDAs digested with collagenase type I, II, and IV (all at $100 \mathrm{U} / \mathrm{ml}$, Worthington) and DNase I (100 U/ml, Worthington). Cells were incubated with Fc receptor blocker (eBioscience, catalog 14-0161-85) followed by staining with the following fluorescence-conjugated antibodies: PE/Cy7 anti-mouse CD45 (catalog 103114), PE anti-mouse CD90.2 (catalog 140308), and APC Streptavidin (catalog 405207) (Biolegend). Biotinylated polyclonal sheep anti-human FAP antibody that cross-reacts with mouse FAP (catalog BAF3715) and biotinylated normal sheep IgG control (catalog BAF020) were purchased from R\&D Systems. Dead cells were excluded by using propidium iodide. Cell acquisition was performed on LSR-II using FACSDiva software (BD Bioscience). Data were analyzed using FlowJo (Tree Star).

Statistics. Statistics were calculated using GraphPad Prism 6 and SPSS Statistics 22. For studies comparing two groups, 2-tailed Student's $t$ test was used. For survival and tumor-detection studies, Mantle-Cox 
(log-rank) test was performed. For studies comparing outcomes between two or more groups, Fisher's exact test or $\chi^{2}$ test was used. Differences were considered significant at $P<0.05$.

Study approval. Experimental studies using animals were approved by the University of Pennsylvania Institutional Animal Care and Use Committee (protocol 805004) and were in compliance with guidelines for the care and use of animals of the NIH. Studies using patient samples were approved by the Institutional Review Board of Taipei Veterans General Hospital.

\section{Author contributions}

$\mathrm{AL}$ and EP conceived and designed the study and wrote the manuscript. AL, RB, PG, DA, and JM conducted experiments, acquired data, and analyzed data. CPL and MH constructed human PDA tissue microarrays and performed staining. ELB (a board-certified veterinary pathologist) analyzed mouse pancreatic tumor pathology.

\section{Acknowledgments}

We thank Ben Stanger and Nicole Aiello for providing KPC-Rosa ${ }^{\text {YFP }}$ mice. We thank James Marx for his clinical care of laboratory mice as well as the histology facility at the Ryan Hospital of the School of Veterinary Medicine at the University of Pennsylvania. We thank Michele Jacob and Leslie Todd who generated the FAP-luciferase reporter mice. This work was supported by grants from the National Cancer Institute and NIH (R21 CA169741, R01 CA141144, and R01 CA 172921 to EP), Academia Sinica, and the Ministry of Science and Technology (MOST 106-0210-01-15-02 to MH). AL was sponsored by a Student Training and Research in Tumor Immunology fellowship from the Cancer Research Institute.

Address correspondence to: Ellen Puré, Hill Pavilion Room 410E, 380 S. University Avenue, Philadelphia, Pennsylvania 19104, USA. Phone: 215.573.9406; Email: epure@upenn.edu.

AL's present address is: Department of Bioengineering, Stanford University, Stanford, California, USA.

1. Ryan DP, Hong TS, Bardeesy N. Pancreatic adenocarcinoma. N Engl J Med. 2014;371(11):1039-1049.

2. Hingorani SR, et al. Trp53R172H and KrasG12D cooperate to promote chromosomal instability and widely metastatic pancreatic ductal adenocarcinoma in mice. Cancer Cell. 2005;7(5):469-483.

3. Ying H, et al. Genetics and biology of pancreatic ductal adenocarcinoma. Genes Dev. 2016;30(4):355-385

4. Knopf JD, Tholen S, Koczorowska MM, De Wever O, Biniossek ML, Schilling O. The stromal cell-surface protease fibroblast activation protein- $\alpha$ localizes to lipid rafts and is recruited to invadopodia. Biochim Biophys Acta. 2015;1853(10 Pt A):2515-2525.

5. Park JE, Lenter MC, Zimmermann RN, Garin-Chesa P, Old LJ, Rettig WJ. Fibroblast activation protein, a dual specificity serine protease expressed in reactive human tumor stromal fibroblasts. J Biol Chem. 1999;274(51):36505-36512.

6. Edosada CY, et al. Peptide substrate profiling defines fibroblast activation protein as an endopeptidase of strict Gly(2)-Pro(1)cleaving specificity. FEBS Lett. 2006;580(6):1581-1586.

7. Wang H, et al. Downregulation of FAP suppresses cell proliferation and metastasis through PTEN/PI3K/AKT and Ras-ERK signaling in oral squamous cell carcinoma. Cell Death Dis. 2014;5:e1155.

8. Kraman M, et al. Suppression of antitumor immunity by stromal cells expressing fibroblast activation protein-alpha. Science. 2010;330(6005):827-830.

9. Santos AM, Jung J, Aziz N, Kissil JL, Puré E. Targeting fibroblast activation protein inhibits tumor stromagenesis and growth in mice. J Clin Invest. 2009;119(12):3613-3625.

10. Keane FM, Nadvi NA, Yao TW, Gorrell MD. Neuropeptide Y, B-type natriuretic peptide, substance P and peptide YY are novel substrates of fibroblast activation protein- $\alpha$. FEBS J. 2011;278(8):1316-1332.

11. Christiansen VJ, Jackson KW, Lee KN, McKee PA. Effect of fibroblast activation protein and alpha2-antiplasmin cleaving enzyme on collagen types I, III, and IV. Arch Biochem Biophys. 2007;457(2):177-186.

12. Koczorowska MM, et al. Fibroblast activation protein- $\alpha$, a stromal cell surface protease, shapes key features of cancer associated fibroblasts through proteome and degradome alterations. Mol Oncol. 2016;10(1):40-58.

13. Yang X, et al. FAP promotes immunosuppression by cancer-associated fibroblasts in the tumor microenvironment via STAT3CCL2 signaling. Cancer Res. 2016;76(14):4124-4135.

14. Baird SK, Allan L, Renner C, Scott FE, Scott AM. Fibroblast activation protein increases metastatic potential of fibrosarcoma line HT1080 through upregulation of integrin-mediated signaling pathways. Clin Exp Metastasis. 2015;32(5):507-516.

15. Ramirez-Montagut T, et al. FAPalpha, a surface peptidase expressed during wound healing, is a tumor suppressor. Oncogene. 2004;23(32):5435-5446.

16. Özdemir BC, et al. Depletion of carcinoma-associated fibroblasts and fibrosis induces immunosuppression and accelerates pancreas cancer with reduced survival. Cancer Cell. 2014;25(6):719-734.

17. Rhim AD, et al. Stromal elements act to restrain, rather than support, pancreatic ductal adenocarcinoma. Cancer Cell. 2014;25(6):735-747.

18. Rhim AD, et al. EMT and dissemination precede pancreatic tumor formation. Cell. 2012;148(1-2):349-361. 
19. Whatcott CJ, et al. Desmoplasia in primary tumors and metastatic lesions of pancreatic cancer. Clin Cancer Res. 2015;21(15):3561-3568.

20. Hwang RF, et al. Cancer-associated stromal fibroblasts promote pancreatic tumor progression. Cancer Res. 2008;68(3):918-926.

21. Xu Z, et al. Role of pancreatic stellate cells in pancreatic cancer metastasis. Am J Pathol. 2010;177(5):2585-2596.

22. Ikenaga N, et al. CD10+ pancreatic stellate cells enhance the progression of pancreatic cancer. Gastroenterology. 2010;139(3):1041-1051.

23. Lo A, et al. Tumor-promoting desmoplasia is disrupted by depleting fap-expressing stromal cells. Cancer Res. 2015;75(14):2800-2810.

24. Feig C, et al. Targeting CXCL12 from FAP-expressing carcinoma-associated fibroblasts synergizes with anti-PD-L1 immunotherapy in pancreatic cancer. Proc Natl Acad Sci USA. 2013;110(50):20212-20217.

25. Cohen SJ, et al. Fibroblast activation protein and its relationship to clinical outcome in pancreatic adenocarcinoma. Pancreas. 2008;37(2):154-158

26. Lee HO, Mullins SR, Franco-Barraza J, Valianou M, Cukierman E, Cheng JD. FAP-overexpressing fibroblasts produce an extracellular matrix that enhances invasive velocity and directionality of pancreatic cancer cells. BMC Cancer. 2011;11:245.

27. Kawase T, et al. Fibroblast activation protein- $\alpha$-expressing fibroblasts promote the progression of pancreatic ductal adenocarcinoma. BMC Gastroenterol. 2015;15:109.

28. Wang LC, et al. Targeting fibroblast activation protein in tumor stroma with chimeric antigen receptor $\mathrm{T}$ cells can inhibit tumor growth and augment host immunity without severe toxicity. Cancer Immunol Res. 2014;2(2):154-166.

29. Tran E, et al. Immune targeting of fibroblast activation protein triggers recognition of multipotent bone marrow stromal cells and cachexia. J Exp Med. 2013;210(6):1125-1135.

30. Schuberth PC, et al. Treatment of malignant pleural mesothelioma by fibroblast activation protein-specific re-directed T cells. J Transl Med. 2013;11:187.

31. Kakarla S, et al. Antitumor effects of chimeric receptor engineered human T cells directed to tumor stroma. Mol Ther. 2013;21(8):1611-1620.

32. Proost $\mathrm{P}$, et al. Processing by CD26/dipeptidyl-peptidase IV reduces the chemotactic and anti-HIV-1 activity of stromal-cell-derived factor-1alpha. FEBS Lett. 1998;432(1-2):73-76. 